\title{
The Identifiability of Covarion Models in Phylogenetics
}

\author{
Elizabeth S. Allman, John A. Rhodes
}

\begin{abstract}
Covarion models of character evolution describe inhomogeneities in substitution processes through time. In phylogenetics, such models are used to describe changing functional constraints or selection regimes during the evolution of biological sequences. In this work the identifiability of such models for generic parameters on a known phylogenetic tree is established, provided the number of covarion classes does not exceed the size of the observable state space. 'Generic parameters' as used here means all parameters except possibly those in a set of measure zero within the parameter space. Combined with earlier results, this implies both the tree and generic numerical parameters are identifiable if the number of classes is strictly smaller than the number of observable states.
\end{abstract}

Index Terms-phylogenetics, Markov processes on trees, covarion models, statistical consistency

\section{INTRODUCTION}

Phylogenetic inference is now generally performed in a statistical framework, using probabilistic models of the evolution of biological sequences, such as DNA or proteins. To rigorously establish the validity of such an approach, a fundamental question that must be addressed is whether the models in use are identifiable: From the theoretical distribution predicted by the model, is it possible to uniquely determine all parameters? Parameters for simple models include the topology of the evolutionary tree, edge lengths on the tree, and rates of various types of substitution, though more complicated models have additional parameters as well. If a model is nonidentifiable, one cannot show that performing inference with it will be statistically consistent. Informally, even with large amounts of data produced by an evolutionary process that was accurately described by the model, we might make erroneous inferences if we use a non-identifiable model.

Identifiability for the most basic phylogenetic models, such as the Jukes-Cantor, Kimura, and all other time-reversible models, follows from Chang's work on the general Markov model [5]. However, for models with rate variation across sites, where the distribution of rates is not fully known, only recently have the first positive results been obtained [2], [3], [1]. Despite its widespread use in data analysis, identifiability of the GTR $+\Gamma+\mathrm{I}$ model has yet to be addressed rigorously. (Unfortunately the proof of identifiability given in [17] has fundamental gaps, as explained in the appendix of [1].)

The covarion model, introduced in its basic mathematical form by Tuffley and Steel [19], incorporates rate variation

Department of Mathematics and Statistics, University of Alaska Fairbanks, PO Box 756660, Fairbanks, AK 99775-6660; e.allman@uaf.edu, j.rhodes@uaf.edu

The authors thank the Isaac Newton Institute and the National Science Foundation. Parts of this work were conducted during residencies at INI, and with support from NSF grant DMS 0714830. within lineages rather than across sites. Extensions of the basic version of the model have appeared in a variety of analyses of experimental data, with authors referring to the model using terminology such as 'covarion' [12], 'covarion-like' [7], [20], 'site-specific rate variation' [7], [10], 'Markov-modulated Markov process' [8], [9], or 'temporal hidden Markov models' [21]. We use the name 'covarion' in this paper for simplicity, although we acknowledge the model does not capture the full complexity of the process originally proposed by Fitch and Markowitz [6]. Informally, the covarion model allows several classes (e.g., invariable, slow, and fast), with characters evolving so they not only change between observable states, but also between classes. Though the class is never observed, it affects the evolutionary process over time. The model thus attempts to capture the fact that substitution rates may speed up or slow down at different sites in a sequence at different times in their descent. Changing functional constraints or selection regimes are possible sources of such a process.

Identifiability of even the tree parameter under the covarion model was not established with its introduction in [19], despite strong efforts. In [2], the authors established that for generic choices of covarion parameters tree topologies are indeed identifiable, provided the number of covarion classes is less than the number of observable states. Thus for nucleotide models of DNA there can be 3 classes, though for amino acid models of proteins one can allow 19 classes, and for codon models of DNA up to 60 classes. 'Generic' here means that there could be some parameter choices for which identifiability fails, though they will be rare (of Lebesgue measure zero). In fact, if parameters are chosen randomly, with any natural notion of random, one can be sure the tree topology is identifiable.

Since the notion of generic identifiability is perhaps not widely known, and will play a key role in this work as well, we elaborate on its meaning. For statistical models in general, it is most desirable to establish identifiability over the full parameter space. However, such a strong claim may not hold, so that the best possible result is to establish identifiability over most of the parameter space, and completely characterize all those parameter choices for which identifiability fails. Generic identifiability results are a little weaker than this, in that while identifiability is established over most of the parameter space, they allow for ignorance about identifiability on a small subset of the parameter space. This exceptional subset of parameter space contains all parameters for which identifiability fails, but may also contain some parameters that are identifiable. Complex statistical models can be quite difficult to analyze, so that generic identifiability is sometimes the strongest known 
result. For instance, though hidden Markov models are widely used in bioinformatics and other fields, and generic identifiability was proved for HHMs in [16], we know of no improvements on that work in the nearly 40 years since it appeared. Phylogenetic models are similar to HMMs in that they posit unobserved variables, at the internal nodes of a tree, but typically have more complex parameterizations than HMMs. Thus we consider their analysis to be even more challenging.

The question of identifiability of numerical parameters for the covarion model was left open by [2]. In this article, we assume the tree topology is known, and establish identifiability of the numerical parameters of several variants of the covarion model for generic parameter choices, provided the number of covarion classes is strictly less than the number of observable states. For certain versions of a covarion model, this can be strengthened to allow one more class, so that the number of classes and observable states may be the same.

We consider three variants of the covarion model, which extend the Tuffley-Steel model, and have previously appeared in works of others, though without our formal terminology: The scaled covarion model, $\mathrm{sCov}$, assumes all classes undergo substitutions according to a common process but at rescaled rates. The equal stationary distribution covarion model, eCov, generalizes this to allow in-class substitution processes to vary more across classes, provided they have identical stationary distributions and class change rates are independent of the base. Finally, in the general covarion model, Cov, each class may undergo substitutions quite differently as long as the entire process is time reversible. Cov is the model described in [21], eCov is developed in [9], and sCov is used in [10].

Note these models are nested,

$$
\mathrm{sCov} \subset \mathrm{eCov} \subset \mathrm{Cov},
$$

though each submodel is non-generic within its supermodels. Because identifiability is established here only for generic parameters, it is necessary to state and prove the generic identifiability of all three covarion models to encompass the range of models used in practice.

In Section III we formally present these models, and in Section [III we state our results precisely. That section also provides an overview of the proof. For those whose primary interest is understanding the result, and who do not wish to delve into the full mathematical arguments behind it, we suggest that reading through Section [III may suffice. The remainder of the paper provides the rather detailed arguments that are essential to rigorously establishing identifiability.

We also note that many practitioners have conducted data analysis with models combining covarion features with acrosssite rate variation, such as that modeled by a discrete $\Gamma$ distribution. While the identifiability of such models has not been established rigorously as of yet, we view the main theorems of this paper as providing a first step toward understanding of these more complex models.

This work was influenced by many useful discussions concerning covarion models that we had with participants of the Isaac Newton Institute's Programme in Phylogenetics.
Simon Whelan deserves particular thanks for explaining his forthcoming work [21].

We also thank the referees for their helpful suggestions, and especially Christopher Tuffley, who noted a flaw in an earlier version of Section VI and suggested the simpler argument that appears there now.

\section{The Parameterization of the Covarion Models}

For the purpose of orientation, we briefly recall a simpler phylogenetic model, the $\kappa$-state general time reversible (GTR) model. The basic state change process is specified by a $\kappa \times \kappa$ rate matrix $Q$, whose off-diagonal $i, j$-entry gives an instantaneous rate $(>0)$ at which a character in state $i$ enters state $j$. Each row of $Q$ must add to 0 . As a consequence, $Q$ has a unique left eigenvector $\pi$ with eigenvalue 0 , the stationary vector for $Q$. Time reversibility is mathematically formulated as the assumption that $\operatorname{diag}(\boldsymbol{\pi}) Q$ is symmetric. Character change along a rooted metric tree $T$ is then modeled as follows: The entries of $\boldsymbol{\pi}$ give the probability that a character is in the various states at the root of the tree. Along each edge $e$ of $T$, directed away from the root, the conditional probabilities of state changes are given by the Markov matrix $M_{e}=\exp \left(Q t_{e}\right)$, where $t_{e} \geq 0$ is the edge length. From this information one can compute the probability of any specification of states at the leaves of the tree. Due to the time reversibility assumption, the location of the root within the tree actually has no effect on this probability distribution. Thus the parameters of the model are the topology of the unrooted tree $T$, the collection of edge lengths $\left\{t_{e}\right\}$, and the rate matrix $Q$.

To present the covarion models, we first focus on the process of state change. It will be convenient to adopt terminology most appropriate to nucleotide sequences. In particular, in discussing covarion models we limit our use of the word 'state' which is commonly used for all Markov models, because the number of states at internal nodes of a tree differs from that at leaves, even though there is a relationship between them. We instead refer to observable states as 'bases,' and to rate classes as 'classes.' Thus at a leaf a state is simply a base, while at an internal node a state is a pair of a class and a base. We caution the reader that this usage of 'base' is not standard in biology, as it encompasses the 4 bases in nucleotide sequences, as well as the 20 amino acids of protein sequences, and the 61 codons in a model of codon substitution. Also, while it is often natural to think of 'classes' as being associated to rate scalings, this may be misleading, as several of the models we formalize allow for more generality. We use $[\kappa]=\{1,2, \ldots, \kappa\}$ to denote the set of bases and $[c]=\{1,2, \ldots, c\}$ to denote the set of classes.

To refer to entries of vectors and matrices of size $c \kappa$, it will be convenient to index entries using interchangeably the set $[c \kappa]$, and the set $[c] \times[\kappa]$ with lexicographic order. Thus the index $(i, j)$, which should be interpreted as the "class $i$, base $j$ ' index, is equivalent to $(i-1) c+j$. Entries in a $c \kappa \times c \kappa$ matrix, then, can be referred to by an ordered pair of indices, each of which is an ordered pair in $[c] \times[\kappa]$.

Let $c, \kappa$ be positive integers. The most general $c$-class, $\kappa$-base covarion model, introduced by Whelan in [21], is specified in the following way: 
(1) For each $i \in[c]$, a base-change process for class $i$ is described by a rate-matrix $Q_{i}$ of size $\kappa \times \kappa$. We assume all $Q_{i}$ are distinct, so that no two classes undergo substitutions at the same rates. For $c-1$ values of $i$ we require that the off-diagonal entries of $Q_{i}$ are strictly positive so that all substitutions are possible, and the rows sum to 0 . For the remaining $Q_{i}$ we only require that all off-diagonal entries be non-negative and that rows sum to 0 . In particular, we allow $Q_{i}$ for at most one $i$ to be the 0-matrix, in order to model an invariable class.

(2) For each ordered pair of classes $i_{1} \neq i_{2}$, a diagonal matrix $S_{i_{1} i_{2}}$ of size $\kappa \times \kappa$ describes switching rates from class $i_{1}$ to class $i_{2}$. The entries of $S_{i_{1} i_{2}}$ are non-negative. The requirement that $S_{i_{1} i_{2}}$ be diagonal will imply that instantaneous base switches do not occur simultaneously with class switches.

(3) Let $R$ be the $c \kappa \times c \kappa$ matrix which, when viewed in $c \times c$ block form, has as its off-diagonal $i_{1}, i_{2}$-block $S_{i_{1} i_{2}}$ and as its $i$ th diagonal block $Q_{i}-\sum_{i_{2}} S_{i i_{2}}$. Note each row of $R$ sums to 0 . We require that $R$ describe a time-reversible process; that is, for some vector $\boldsymbol{\mu}$ with positive entries summing to 1 the matrix

$$
\operatorname{diag}(\boldsymbol{\mu}) R
$$

is symmetric.

We may rescale $R$, or equivalently all entries of the $Q_{i}$ and $S_{i_{1} i_{2}}$, so that

$$
\operatorname{trace}(\operatorname{diag}(\boldsymbol{\mu}) R)=-1 .
$$

Requiring this normalization avoids a trivial non-identifiability issue in which rescaling of edge lengths would have the same effect as rescaling $R$. It also imposes a scale on edge lengths so that the average instantaneous rate of (base,class) changes under the Markov process is 1 per unit of edge length. We will assume throughout the rest of this paper that this normalization has been made. Consequently, if two such matrices are multiples of one another, we may conclude they are equal.

Any matrix $R$ with these properties will be called a covarion rate matrix for the general covarion model, $\operatorname{Cov}(c, \kappa)$, with $c$ classes and $\kappa$ bases.

We may write

$$
\boldsymbol{\mu}=\left(\sigma_{1} \boldsymbol{\pi}_{1}, \sigma_{2} \boldsymbol{\pi}_{2}, \ldots, \sigma_{c} \boldsymbol{\pi}_{c}\right)
$$

where the $\boldsymbol{\pi}_{i} \in \mathbb{R}^{\kappa}$ and $\boldsymbol{\sigma}=\left(\sigma_{1}, \ldots, \sigma_{c}\right) \in \mathbb{R}^{c}$ are vectors of positive entries summing to 1 . Then the symmetry of $\operatorname{diag}(\boldsymbol{\mu}) R$ implies the symmetry of $\operatorname{diag}\left(\boldsymbol{\pi}_{i}\right) Q_{i}$ for each $i$. Thus our assumptions ensure the $Q_{i}$ each define timereversible processes. Additionally we find

$$
\sigma_{i_{1}} \operatorname{diag}\left(\boldsymbol{\pi}_{i_{1}}\right) S_{i_{1} i_{2}}=\sigma_{i_{2}} \operatorname{diag}\left(\boldsymbol{\pi}_{i_{2}}\right) S_{i_{2} i_{1}} .
$$

These conditions are equivalent to the time-reversibility of $R$.

A specialization of $\operatorname{Cov}(c, \kappa)$ described in [9] assumes further that

(4) The base substitution processes described by the $Q_{i}$ have equal stationary distributions, $\boldsymbol{\pi}_{i}=\boldsymbol{\pi}$.

(5) The switching matrices $S_{i_{1} i_{2}}$ are scalar, so $S_{i_{1} i_{2}}=$ $s_{i_{1} i_{2}} I_{\kappa}$, where $I_{\kappa}$ is the $\kappa \times \kappa$ identity matrix.

We refer to this as the equal stationary distribution covarion model, denoted by $\operatorname{eCov}(c, \kappa)$.

The model $\operatorname{eCov}(c, \kappa)$ can also be conveniently described in tensor notation. For any vectors or matrices $A=\left(a_{i_{1} i_{2}}\right)$ and $B=\left(b_{j_{1} j_{2}}\right)$, let $A \otimes B$ denote the tensor, or Kronecker, product. Using ordered-pair indices as above, we order rows and columns of $A \otimes B$ so the $\left(i_{1}, j_{1}\right),\left(i_{2}, j_{2}\right)$ entry is $a_{i_{1} i_{2}} b_{j_{1} j_{2}}$. With the class switching process for eCov specified by a $c \times c$ rate matrix $S$ with off-diagonal entries $s_{i_{1} i_{2}}$, and rows summing to 0 , then

$$
\begin{gathered}
R=\operatorname{diag}\left(Q_{1}, Q_{2}, \ldots, Q_{c}\right)+S \otimes I_{\kappa}, \\
\boldsymbol{\mu}=\boldsymbol{\sigma} \otimes \boldsymbol{\pi} .
\end{gathered}
$$

The symmetry of $\operatorname{diag}(\boldsymbol{\mu}) R$ is equivalent to the symmetry of each $\operatorname{diag}(\boldsymbol{\pi}) Q_{i}$ and of $\operatorname{diag}(\boldsymbol{\sigma}) S$. Thus the class switching process described by $S$ is time-reversible as well.

A further specialization from eCov yields the scaled covarion model, $\mathrm{s} \operatorname{Cov}(c, \kappa)$, which assumes

(6) For some rate matrix $Q$ and distinct non-negative $r_{1}, r_{2}, \ldots, r_{c}, Q_{i}=r_{i} Q$.

For this submodel, the full covarion process has rate matrix

$$
R=\operatorname{diag}\left(r_{1}, r_{2}, \ldots, r_{c}\right) \otimes Q+S \otimes I_{\kappa} .
$$

Example 1: $\operatorname{sCov}(2,4)$ is just a generalization of the Tuffley-Steel covarion model of nucleotide substitution [19]. For any $s_{1}, s_{2}>0$, let

$S=\left(\begin{array}{cc}-s_{1} & s_{1} \\ s_{2} & -s_{2}\end{array}\right), \quad \boldsymbol{\sigma}=\left(\sigma_{1}, \sigma_{2}\right)=\left(\frac{s_{2}}{s_{1}+s_{2}}, \frac{s_{1}}{s_{1}+s_{2}}\right)$.

Then $S$ defines a time-reversible switching process with stationary vector $\boldsymbol{\sigma}$. For any $Q, \boldsymbol{\pi}$ of a 4-base GTR model, taking $1=r_{1}>r_{2}$ we obtain a rate matrix with block structure

$$
\lambda\left(\begin{array}{cc}
Q-s_{1} I & s_{1} I \\
s_{2} I & r_{2} Q-s_{2} I
\end{array}\right),
$$

while

$\boldsymbol{\mu}=\left(\sigma_{1} \pi_{1}, \sigma_{1} \pi_{2}, \sigma_{1} \pi_{3}, \sigma_{1} \pi_{4}, \sigma_{2} \pi_{1}, \sigma_{2} \pi_{2}, \sigma_{2} \pi_{3}, \sigma_{2} \pi_{4}\right)$.

If $r_{2}=0$, then an invariable class is included, and this is exactly the Tuffley-Steel model.

Example 2: If $c \geq 3$, the requirement for $\operatorname{eCov}(c, \kappa)$ that the class switching process described by $S$ be time-reversible implies stronger relationships among its entries than merely requiring rows sum to 0 . If

$$
S=\left(\begin{array}{ccc}
-\left(s_{12}+s_{13}\right) & s_{12} & s_{13} \\
s_{21} & -\left(s_{21}+s_{23}\right) & s_{23} \\
s_{31} & s_{32} & -\left(s_{31}+s_{32}\right)
\end{array}\right),
$$

and $\boldsymbol{\sigma}$ are such that $\operatorname{diag}(\boldsymbol{\sigma}) S$ is symmetric, then one can show (most easily by using symbolic algebra software, such as Maple or Singular) that

$$
s_{12} s_{23} s_{31}-s_{13} s_{21} s_{32}=0,
$$

and

$$
\boldsymbol{\sigma}=\frac{1}{s_{21} s_{32}+s_{12} s_{32}+s_{12} s_{23}}\left(s_{21} s_{32}, s_{12} s_{32}, s_{12} s_{23}\right) .
$$


Let $Q_{1}, Q_{2}, Q_{3}$ denote $\kappa$-base GTR rate matrices with a common stationary vector $\pi$. Then, up to a scaling factor, the matrix

$$
\left(\begin{array}{ccc}
Q_{1}-\left(s_{12}+s_{13}\right) I & s_{12} I & s_{13} I \\
s_{21} I & Q_{2}-\left(s_{21}+s_{23}\right) I & s_{23} I \\
s_{31} I & s_{32} I & Q_{3}-\left(s_{31}+s_{32}\right) I
\end{array}\right)
$$

is a rate matrix for $\operatorname{eCov}(3, \kappa)$ with stationary vector

$$
\boldsymbol{\mu}=\left(\begin{array}{lll}
\sigma_{1} \boldsymbol{\pi} & \sigma_{2} \boldsymbol{\pi} & \sigma_{3} \boldsymbol{\pi}
\end{array}\right) .
$$

Such models are presented in [9].

Example 3: Let $Q_{1}, Q_{2}$ denote $\kappa$-base GTR rate matrices, with stationary vectors $\boldsymbol{\pi}_{1}, \boldsymbol{\pi}_{2}$. Let $\boldsymbol{\sigma}=\left(\sigma_{1}, \sigma_{2}\right)$ be any vector of positive entries summing to 1 , and $s=\left(s_{1}, s_{2}, \ldots, s_{\kappa}\right)$ any vector of positive numbers. Then defining

$$
\begin{aligned}
& S_{12}=\sigma_{2} \operatorname{diag}\left(\boldsymbol{\pi}_{2}\right) \operatorname{diag}(\boldsymbol{s}), \\
& S_{21}=\sigma_{1} \operatorname{diag}\left(\boldsymbol{\pi}_{1}\right) \operatorname{diag}(\boldsymbol{s}),
\end{aligned}
$$

ensures that equation (1) is satisfied. For suitable $\lambda$, the matrix

$$
\lambda\left(\begin{array}{cc}
Q_{1}-S_{12} & S_{12} \\
S_{21} & Q_{2}-S_{21}
\end{array}\right)
$$

is thus a rate matrix for the model $\operatorname{Cov}(2, \kappa)$, and of the type described in [21].

To specify any of the covarion models $\operatorname{Cov}(c, \kappa)$, $\mathrm{eCov}(c, \kappa)$, or $\operatorname{sCov}(c, \kappa)$ on a topological tree $T$, in addition to $R$ we must specify edge lengths $\left\{t_{e}\right\}$. These determine Markov matrices $M_{e}$ for each edge $e$ of the tree as follows: For every internal edge $e$ of the tree, $M_{e}=\exp \left(R t_{e}\right)$ is $c \kappa \times c \kappa$ and describes (class, base)-substitutions over the edge. Letting $\mathbf{1}_{c}=\left(\begin{array}{llll}1 & 1 & \ldots & 1\end{array}\right) \in \mathbb{R}^{c}$ be a row vector, and $I_{\kappa}$ the $\kappa \times \kappa$ identity, set

$$
J=\mathbf{1}_{c}^{T} \otimes I_{\kappa}=\left(\begin{array}{llll}
I_{\kappa} & I_{\kappa} & \ldots & I_{\kappa}
\end{array}\right)^{T} .
$$

Then on every pendant edge $e$ of the tree, $M_{e}=\exp \left(R t_{e}\right) J$ is $c \kappa \times \kappa$. Notice that $J$ serves to hide class information, by summing over it, so that only bases may be observed.

Because the process defined by $R$ is reversible, we may arbitrarily choose any internal vertex of the tree as the root, and using $\boldsymbol{\mu}$ as a root distribution compute the joint distribution of bases at the leaves of the tree in the usual way for Markovian phylogenetic models on trees. For an $n$-leaf tree, this distribution is naturally thought of as an $n$-dimensional $\kappa \times \kappa \times \cdots \times \kappa$ array.

Let $P=\hat{P} \otimes I_{\kappa}$, where $\hat{P}$ is a $c \times c$ permutation matrix. Then replacing $R$ by $P^{T} R P$ simply permutes the classes. As no information on classes is observed, it is easy to see this has no effect on the joint distribution of bases arising from a covarion model. Thus we must account for this trivial source of non-identifiability. For $\operatorname{sCov}(c, \kappa)$ this could be done by requiring the $r_{i}$ be enumerated in descending order. However, for $\operatorname{Cov}(c, \kappa)$ and $\operatorname{eCov}(c, \kappa)$ there need not be any natural ordering of the $Q_{i}$. To treat all these models uniformly, we will seek identifiability only up to permutation of classes.

Note that as formulated above, the covarion models generalize mixture models on a single tree with a finite number of classes. Indeed, one need only choose the switching matrix $S$ for sCov or eCov to be the zero matrix, or set all $S_{i_{1} i_{2}}=0$ for Cov, to describe across-site rate variation. However, such choices are non-generic - of Lebesgue measure zero within the covarion models. Since our main result allows for nongeneric exceptions to identifiability, we caution that it does not rigorously imply anything about across-site rate variation models, though it is perhaps suggestive.

At one point in our arguments we will in fact need an assumption that rules out consideration of across-site rate variation models. In Lemma 12, we require that the switching process for $\operatorname{Cov}(c, \kappa)$ is irreducible in the following sense: Say class $i$ communicates to class $i^{\prime}$ when all diagonal entries of $S_{i i^{\prime}}$ are positive. Then class irreducibility of $R$ will mean that for each pair of classes $i \neq i^{\prime}$ there is a chain of classes $i=i_{0}, i_{1}, i_{2}, \ldots, i_{n}=i^{\prime}$ with $i_{k}$ communicating to $i_{k+1}$. For the models eCov and $\mathrm{sCov}$, this definition is equivalent to the usual definition of irreducibility, [11], for the Markov process described by the switching matrix $S$. Moreover, class irreducibility of $R$, together with the assumption that all entries of some $Q_{i}$ are non-zero implies irreducibility of $R$ in the usual sense.

Note that class irreducibility holds for generic choices of covarion parameters for all three covarion models, as generically all diagonal entries of all $S_{i i^{\prime}}$ are non-zero. Therefore, despite its important role in establishing the results, we do not refer to irreducibility explicitly in statements of theorems which only make claims for generic parameter choices.

\section{Statement of TheOREMS AND OVERVIEW}

We establish the following:

Theorem 1: Consider the models $\operatorname{Cov}(c, \kappa), \operatorname{eCov}(c, \kappa)$, and $\operatorname{sCov}(c, \kappa)$ on an $n$-leaf binary tree, $n \geq 7$. If the tree topology is known, then for generic choices of parameters all numerical parameters are identifiable, up to permutation of classes, provided $c \leq \kappa$ for $\mathrm{sCov}$ and $\mathrm{eCov}$, and provided $c<\kappa$ for Cov.

Combined with earlier work in [2], this shows:

Corollary 2: Consider the models $\operatorname{Cov}(c, \kappa), \operatorname{eCov}(c, \kappa)$, and $\operatorname{sCov}(c, \kappa)$ on an $n$-leaf binary tree, $n \geq 7$. Then for generic choices of parameters, the tree topology and all numerical parameters are identifiable, up to permutation of classes, provided $c<\kappa$.

In outline, the proof of the theorem is as follows: Section IV addresses basic properties of eigenvectors and eigenvalues of a covarion rate matrix, and discusses the form of joint distributions from covarion models on 2-leaf trees. This section provides preliminary results needed for the main arguments, which span the remainder of this article.

To establish identifiability of model parameters on a particular tree, our argument will require that there be a 6-leaf subtree with the particular topology shown in Figure 1. It is easy to see that any tree with at least 7 leaves contains such a 6-leaf subtree. (For simplicity, we chose to state Theorem 1 and its corollary for trees of 7 or more taxa, even though they also hold for this 6-leaf tree.) 


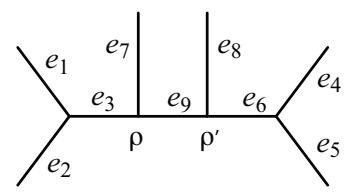

Fig. 1. The 6-leaf tree on which arguments will be based, with edges $e_{i}$ and internal nodes $\rho, \rho^{\prime}$.

In Section $\mathrm{V}$ the main thread of the proof begins. We use algebraic arguments built on a theorem of J. Kruskal [15] to determine the covarion Markov matrix $M=\exp \left(R t_{9}\right)$ describing the total substitution process over the central edge $e_{9}$, of length $t_{9}$, in the tree of Figure 1 up to permutation of the rows and columns. This part of our argument is not very specific to the covarion model, but rather applies to more general models provided the Markov matrices involved satisfy some technical algebraic conditions. We therefore must show that Markov matrices arising from the covarion model, as exponentials of a covarion rate matrix, satisfy these technical conditions, at least for generic parameter choices. Though this fact is completely plausible, establishing it rigorously requires rather detailed work, which is completed in Section VI This part of our argument is the reason Theorem 1 refers to identifiability of 'generic' parameters and not all parameters, as well as the reason we require $c \leq \kappa$.

Once the Markov matrix on the central edge of the tree is identified up to row and column permutations, to determine the covarion rate matrix we must determine the correct row and column orderings, and take a matrix logarithm. We are able to show there is a unique ordering of rows and columns that produces a covarion rate matrix in part by taking advantage of the pattern of zeros that must appear in such a rate matrix. Other facts about rate matrices, such as the non-positivity of eigenvalues, also play a role. We obtain an essential piece of information on the ordering from the known ordering of bases at the leaves of the tree. All this is the content of Section VII

Finally, once we have determined the covarion rate matrix from this central edge, we use it in Section VIII to determine the sum of edge lengths between any two leaves in the tree. By standard arguments, we may then determine the lengths of all individual edges in the tree, so all parameters have been identified.

Note that the later steps of our arguments are constructive, in that one could apply them to a specific probability distribution to explicitly recover the parameters producing it. However, Kruskal's theorem is not constructive; it guarantees a unique set of parameters but does not indicate a procedure for recovering them. A constructive version of Kruskal's theorem would give an algorithm for the decomposition of threedimensional tensors into minimal sums of rank 1 tensors. This is an interesting but challenging open problem, which would have applications in several other areas of applied mathematics as well. However, the particular case of Kruskal's theorem we use can also be established by a longer argument, which we omit, along the lines of the identifiability result in [5]. Using that approach one obtains an explicit parameter identification procedure that depends on the calculation of eigenvectors for $c \kappa \times c \kappa$ matrices.

\section{DIAGONALIZING COVARION RATE MATRICES}

We summarize a few basic facts concerning the eigenvectors and eigenvalues of a covarion rate matrix $R$, under the hypotheses of the $\operatorname{Cov}(c, \kappa)$ model.

If $R$ is a rate matrix for $\operatorname{Cov}(c, \kappa)$ then it is timereversible by assumption. Thus $\operatorname{diag}(\boldsymbol{\mu}) R$ is symmetric, and $\operatorname{diag}(\boldsymbol{\mu})^{1 / 2} R \operatorname{diag}(\boldsymbol{\mu})^{-1 / 2}$ is as well. Therefore

$$
\operatorname{diag}(\boldsymbol{\mu})^{1 / 2} R \operatorname{diag}(\boldsymbol{\mu})^{-1 / 2}=C^{T} B C
$$

for some orthogonal $C$ and real diagonal $B$. Letting $U=$ $C \operatorname{diag}(\boldsymbol{\mu})^{1 / 2}$, we have

$$
R=U^{-1} B U, \quad U^{-1}=\operatorname{diag}(\boldsymbol{\mu})^{-1} U^{T} .
$$

If $R$ is class irreducible, then it is irreducible. Thus one of its eigenvalues is 0 and the others are strictly negative [11]. We may thus assume $B=\operatorname{diag}\left(\beta_{1}, \beta_{2}, \ldots, \beta_{c \kappa}\right)$, where $0=$ $\beta_{1}>\beta_{2} \geq \cdots \geq \beta_{c \kappa}$ for generic $R$.

Note that for the model $\operatorname{sCov}(c, \kappa)$, much more can be said about this diagonalization. In [8], it is shown that the eigenvectors and eigenvalues for a scaled covarion rate matrix $R$ are related to those of $Q$ and certain modifications of $S$ through a tensor decomposition.

We now investigate the implications of the diagonalization of covarion rate matrices for 2-taxon probability distributions arising from the model. This will be useful for identifying edge lengths in Section VIII

Suppose $R=U^{-1} B U$ is the diagonalization described above. A 2-taxon distribution, arising from edge length $t$, is described by a $\kappa \times \kappa$ matrix

$$
\begin{aligned}
N & =J^{T} \operatorname{diag}(\boldsymbol{\mu}) \exp (R t) J \\
& =J^{T} \operatorname{diag}(\boldsymbol{\mu}) U^{-1} \exp (B t) U J \\
& =J^{T} U^{T} \exp (B t) U J \\
& =(U J)^{T} \exp (B t)(U J) .
\end{aligned}
$$

We formalize this observation with the following lemma.

Lemma 3: Let $R$ be a covarion rate matrix for $\operatorname{Cov}(c, \kappa)$. Then $R$ determines a matrix $B=\operatorname{diag}\left(\beta_{1}, \ldots, \beta_{c \kappa}\right)$ with $0=$ $\beta_{1}>\beta_{2} \geq \cdots \geq \beta_{c \kappa}$, and a rank $\kappa$ matrix $K$ of size $c \kappa \times \kappa$ such that the probability distribution arising from the covarion model with rate matrix $R$ on a one-edge tree of length $t$ is

$$
N=K^{T} \exp (B t) K .
$$

Proof: It only remains to justify that the rank of $K=U J$ is $\kappa$. However, since $U$ is non-singular, $\operatorname{rank} K=\operatorname{rank} J=\kappa$.

\section{IDENTIFYING A MARKOV MATRIX ON THE CENTRAL EDGE}

The basic identifiability result on which we build our later arguments is a theorem of J. Kruskal [15]. (See also [14], [13] for more expository presentations.) 
For $i=1,2,3$, let $N_{i}$ be a matrix of size $r \times \kappa_{i}$, with $\mathbf{n}_{j}^{i}$ the $j$ th row of $N_{i}$. Let $\left[N_{1}, N_{2}, N_{3}\right]$ denote the $\kappa_{1} \times \kappa_{2} \times \kappa_{3}$ tensor defined by

$$
\left[N_{1}, N_{2}, N_{3}\right]=\sum_{j=1}^{r} \mathbf{n}_{j}^{1} \otimes \mathbf{n}_{j}^{2} \otimes \mathbf{n}_{j}^{3} .
$$

Thus the $\left(k_{1}, k_{2}, k_{3}\right)$ entry of $\left[N_{1}, N_{2}, N_{3}\right] \quad$ is $\sum_{j=1}^{r} \mathbf{n}_{j}^{1}\left(k_{1}\right) \mathbf{n}_{j}^{2}\left(k_{2}\right) \mathbf{n}_{j}^{3}\left(k_{3}\right)$, and this 'matrix triple product' can be viewed as a generalization of the product of two matrices (with one matrix transposed).

Note that simultaneously permuting the rows of all the $N_{i}$ (i.e., replacing each $N_{i}$ by $P N_{i}$ where $P$ is an $r \times r$ permutation) leaves $\left[N_{1}, N_{2}, N_{3}\right]$ unchanged. Also rescaling the rows of each $N_{i}$ so that the scaling factors $c_{j}^{i}$ used for the $\mathbf{n}_{j}^{i}, i=1,2,3$ satisfy $c_{j}^{1} c_{j}^{2} c_{j}^{3}=1$ (i.e., replacing each $N_{i}$ by $D_{i} N_{i}$, where $D_{i}$ is diagonal and $D_{1} D_{2} D_{3}=I$ ) also leaves $\left[N_{1}, N_{2}, N_{3}\right]$ unchanged. That under certain conditions these are the only changes leaving $\left[N_{1}, N_{2}, N_{3}\right]$ fixed is the essential content of Kruskal's theorem.

To state the theorem formally requires one further definition. For a matrix $N$, the Kruskal rank of $N$ will mean the largest number $j$ such that every set of $j$ rows of $N$ are independent. Note that this concept would change if we replaced 'row' by 'column,' but we will only use the row version in this paper. With the Kruskal rank of $N$ denoted by $\operatorname{rank}_{K} N$, observe that

$$
\operatorname{rank}_{K} N \leq \operatorname{rank} N
$$

Theorem 4: (Kruskal) Let $j_{i}=\operatorname{rank}_{K} N_{i}$. If

$$
j_{1}+j_{2}+j_{3} \geq 2 r+2,
$$

then $\left[N_{1}, N_{2}, N_{3}\right]$ uniquely determines the $N_{i}$, up to simultaneously permutating and rescaling the rows. That is, if $\left[N_{1}, N_{2}, N_{3}\right]=\left[N_{1}^{\prime}, N_{2}^{\prime}, N_{3}^{\prime}\right]$, then there exists a permutation $P$ and diagonal $D_{i}$, with $D_{1} D_{2} D_{3}=I$, such that $N_{i}^{\prime}=$ $P D_{i} N_{i}$.

We will apply this result to identify parameters of a stochastic model with a hidden variable. In phylogenetic terms, the model is one on a 3-leaf tree, rooted at the central node. A hidden variable at the central node has $r$ states, and observed variables at the leaves have $\kappa_{1}, \kappa_{2}, \kappa_{3}$ states respectively. Markov matrices $M_{i}$, of size $r \times \kappa_{i}$, describe transitions from the state at the central node to those on leaf $i$, with observed variables conditionally independent given the state of the hidden variable. For each $i=1,2,3$, let $\mathbf{m}_{j}^{i}$ denote the $j$ th row of $M_{i}$. One then checks that the joint distribution for such a model is given by

$$
\left[\mathbf{v} ; M_{1}, M_{2}, M_{3}\right]=\sum_{j=1}^{r} v_{j} \mathbf{m}_{j}^{1} \otimes \mathbf{m}_{j}^{2} \otimes \mathbf{m}_{j}^{3} .
$$

Corollary 5: Suppose $M_{i}, i=1,2,3$, are $r \times \kappa_{i}$ Markov matrices, and $\mathbf{v}=\left(v_{1}, \ldots, v_{r}\right)$ is a row vector of non-zero numbers summing to 1 . Let $j_{i}=\operatorname{rank}_{K} M_{i}$. If

$$
j_{1}+j_{2}+j_{3} \geq 2 r+2,
$$

then $\left[\mathbf{v} ; M_{1}, M_{2}, M_{3}\right]$ uniquely determines $\mathbf{v}, M_{1}, M_{2}, M_{3}$ up to permutation. That is, $\left[\mathbf{v} ; M_{1}, M_{2}, M_{3}\right]=\left[\mathbf{v}^{\prime} ; M_{1}^{\prime}, M_{2}^{\prime}, M_{3}^{\prime}\right]$ implies that there exists a permutation $P$ such that $M_{i}^{\prime}=P M_{i}$ and $\mathbf{v}^{\prime}=\mathbf{v} P^{T}$.

Proof: This follows from Kruskal's theorem in a straightforward manner, using that the rows of each Markov matrix $M_{i}$ sum to 1 .

Remark 1: The corollary actually claims identifiability for generic parameters, where 'generic' is used in the sense of algebraic geometry. To see this, note that for any fixed choice of a positive integer $j_{i}$, those matrices $M_{i}$ whose Kruskal rank is strictly less than $j_{i}$ form an algebraic variety. This is because the matrices for which a specific set of $j_{i}$ rows are dependent is the zero set of all $j_{i} \times j_{i}$ minors obtained from those rows. Then, by taking appropriate products of these minors for different sets of rows we may obtain a set of polynomials whose zero set is precisely those matrices of Kruskal rank $<j_{i}$.

To apply the Corollary of Kruskal's theorem in a phylogenetic setting, we need one additional definition. Given matrices $N_{1}$ of size $r \times s$ and $N_{2}$ of size $r \times t$, let

$$
N=N_{1} \otimes^{\text {row }} N_{2}
$$

denote the $r \times s t$ matrix that is obtained from row-wise tensor products. That is, the $i$ th row of $N$ is the tensor product of the $i$ th row of $N_{1}$ and the $i$ th row of $N_{2}$. Although we do not need a specific ordering of the columns of $N$, we could, for instance, define $N$ by $N(i, j+s(k-1))=N_{1}(i, j) N_{2}(i, k)$.

To interpret this row-wise tensor product in the context of models, consider a rooted tree with two leaves, and a Markov model with $r$ states at the root, and $\kappa_{i}$ states at leaf $i, i=$ 1,2 . Then the transition probabilities from states at the root to states at leaf $i$ are specified by an $r \times \kappa_{i}$ matrix $M_{i}$ of non-negative numbers whose rows add to 1 . The matrix $M=$ $M_{1} \otimes^{\text {row }} M_{2}$ will also have non-negative entries, with rows summing to 1 . Its entries give transition probabilities from the $r$ states at the root to the $\kappa_{1} \kappa_{2}$ composite states at the leaves, formed by specifying the state at both leaves. Thus this row tensor operation is essentially what underlies the notion of a 'flattening' of a multidimensional tensor that plays an important role in [4], [2].

Kruskal's result will actually be applied to a model on a 5-leaf tree, by a method we now indicate. For the 5-leaf tree shown in Figure 2, rooted at $\rho$, suppose Markov matrices $\widetilde{M}_{i}$ (not necessarily square) are associated to all edges to describe transition probabilities of states moving away from the root.

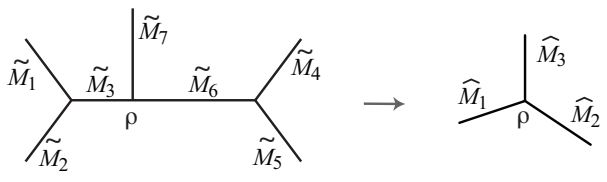

Fig. 2. Viewing a model on a 5-leaf tree as a model on a 3-leaf tree. 
Then with

$$
\begin{aligned}
& \widehat{M}_{1}=\widetilde{M}_{3}\left(\widetilde{M}_{1} \otimes^{\text {row }} \widetilde{M}_{2}\right) \\
& \widehat{M}_{2}=\widetilde{M}_{6}\left(\widetilde{M}_{4} \otimes^{\text {row }} \widetilde{M}_{5}\right) \\
& \widehat{M}_{3}=\widetilde{M}_{7}
\end{aligned}
$$

we obtain Markov matrices on a simpler 3-leaf tree rooted at its central node. Retaining as root distribution the root distribution $\mathbf{v}$ at $\rho$, the joint distribution for this simpler tree is $\left[\mathbf{v} ; \widehat{M}_{1}, \widehat{M}_{2}, \widehat{M}_{3}\right]$. The entries of the distribution for the 5leaf tree and the 3-leaf tree are of course the same, though one is organized as a 5-dimensional array and the other as a 3-dimensional array. However, the reorganization into a 3dimensional array is crucial in allowing us to apply Kruskal's theorem.

Lemma 6: On the 6-leaf tree of Figure 1 rooted at $\rho$, consider a Markov model with $r$ states at all internal nodes and $\kappa$ states at leaves. Let the state distribution at the root be specified by $\mathbf{v}$, and Markov matrices $M_{i}$ describe transitions on edge $e_{i}$ directed away from the root, so for internal edges the $M_{i}$ are $r \times r$, and on pendant edges are $r \times \kappa$.

Suppose in addition

(1) all entries of both $\mathbf{v}$ and $\mathbf{v}^{\prime}=\mathbf{v} M_{9}$ are positive,

(2) the four matrices $M_{6}\left(M_{4} \otimes^{\text {row }} M_{5}\right), M_{9} M_{6}\left(M_{4} \otimes^{\text {row }}\right.$ $\left.M_{5}\right), M_{3}\left(M_{1} \otimes^{\text {row }} M_{2}\right)$, and $M_{9}^{\prime} M_{3}\left(M_{1} \otimes^{\text {row }} M_{2}\right)$, where $M_{9}^{\prime}=\operatorname{diag}\left(\mathbf{v}^{\prime}\right)^{-1} M_{9}^{T} \operatorname{diag}(\mathbf{v})$, all have rank $r$.

(3) the Kruskal ranks of $M_{7}$ and $M_{8}$ are $\geq 2$.

Then $M_{9}, M_{7}$, and $\mathbf{v}$ are uniquely determined from the joint distribution, up to permutation. That is, from the joint distribution we may determine matrices $N_{9}, N_{7}$ and a vector w with $N_{9}=P_{1}^{T} M_{9} P_{2}, N_{7}=P_{1}^{T} M_{7}$, and $\mathbf{w}=\mathbf{v} P_{1}$ for some unknown permutations $P_{1}$ and $P_{2}$.

Proof: Note that since the matrices in (2) have rank $r$, which is equal to the number of their rows, they also have Kruskal rank $r$.

First consider the 5-leaf subtree where edge $e_{8}$ has been deleted, and edges $e_{9}$ and $e_{6}$ conjoined. Then by Corollary 5. we may determine $\mathbf{v} P_{1}$ and the matrices $P_{1}^{T} M_{3}\left(M_{1} \otimes\right.$ row $\left.M_{2}\right), P_{1}^{T} M_{9} M_{6}\left(M_{4} \otimes^{\text {row }} M_{5}\right)$, and $P_{1}^{T} M_{7}$ for some unknown permutation $P_{1}$.

Now reroot the tree of Figure 1 at $\rho^{\prime}$, using root distribution $\mathbf{v}^{\prime}$ and matrix $M_{9}^{\prime}$ on edge $e_{9}$ (directed oppositely), without affecting the joint distribution at the leaves. Having done this, consider the 5-leaf subtree where edge 7 has been deleted. Another application of the corollary determines $\mathbf{v}^{\prime} P_{2}$, $P_{2}^{T} M_{6}\left(M_{4} \otimes^{\text {row }} M_{5}\right), P_{2}^{T} M_{9}^{\prime} M_{3}\left(M_{1} \otimes^{\text {row }} M_{2}\right)$, and $P_{2}^{T} M_{8}$.

Finally, from the $r \times \kappa^{2}$ matrices $A=P_{1}^{T} M_{9} M_{6}\left(M_{4} \otimes\right.$ row $\left.M_{5}\right)$ and $B=P_{2}^{T} M_{6}\left(M_{4} \otimes^{\text {row }} M_{5}\right)$, which by assumption have rank $r$, we may determine the $r \times r$ matrix $C=$ $P_{1}^{T} M_{9} P_{2}$ : since both $A$ and $B$ have rank $r$, the equation $A=C B$ uniquely determines $C$.

Note that for the covarion models, $\mathbf{v}$ has positive entries by assumption, and since $R$ is time reversible with stationary vector $\mathbf{v}$, we will have $\mathbf{v}^{\prime}=\mathbf{v}$ and $M_{9}^{\prime}=M_{9}$. Thus condition (1) will automatically be satisfied in our application of the lemma.

The only potential obstacle to applying Lemma 6 to the covarion model is that we must know that assumptions (2) and (3) on the ranks of various products of Markov matrices are met. While one would certainly suspect that at least for generic choices of covarion parameters there would be no problem, it is non-trivial to establish this rigorously. That is the content of the next lemma.

Let $\left\{f_{1}, \ldots, f_{n}\right\}$ be a finite collection of analytic functions with common domain $D \subseteq \mathbb{C}^{n}$. Recall that the analytic variety $V=V\left(f_{1}, \ldots, f_{n}\right)$ is the subset of $D$ on which all $f_{i}$ vanish. In the next lemma we will use the existence of a single point in $D \backslash V$ to conclude that the $V$ is of strictly lower dimension than $D$. This step may not be familiar to most researchers in phylogenetics, so we recall a simpler instance. A powerful theorem concerning analytic functions of a single complex variable is that if an analytic function $f$ is not identically zero, then any zeros of $f$ in the interior of its domain must be isolated. Equivalently, if there is a single point $z_{0}$ with $f\left(z_{0}\right) \neq 0$, then the zero set of $f$ is a zero-dimensional subset of the one-dimensional domain of $f$. Our argument simply uses a generalization of this fact from the theory of functions of several complex variables.

Lemma 7: Identify the stochastic parameter space $\mathcal{S}$ of any of the models $\operatorname{Cov}(c, \kappa), \operatorname{eCov}(c, \kappa)$ or $\operatorname{sCov}(c, \kappa)$ on the 6taxon tree of Figure 1 with a full-dimensional subset of $\mathbb{R}^{L}$ so that the parameterization map for the probability distribution is given by analytic functions.

Let $X \subset \mathcal{S}$ be the subset on which either at least one of the four $c \kappa \times \kappa^{2}$ matrices arising from cherries,

$$
\begin{gathered}
\exp \left(R t_{3}\right)\left(\exp \left(R t_{1}\right) J \otimes^{\text {row }} \exp \left(R t_{2}\right) J\right), \\
\exp \left(R\left(t_{3}+t_{9}\right)\right)\left(\exp \left(R t_{1}\right) J \otimes^{\text {row }} \exp \left(R t_{2}\right) J\right), \\
\exp \left(R t_{6}\right)\left(\exp \left(R t_{4}\right) J \otimes^{\text {row }} \exp \left(R t_{5}\right) J\right), \\
\exp \left(R\left(t_{6}+t_{9}\right)\right)\left(\exp \left(R t_{4}\right) J \otimes^{\text {row }} \exp \left(R t_{5}\right) J\right),
\end{gathered}
$$

has rank $<c \kappa$, or at least one of the two matrices

$$
\exp \left(R t_{7}\right) J, \quad \exp \left(R t_{8}\right) J
$$

on the pendant edges $e_{7}, e_{8}$ has Kruskal rank $<2$. Then if $c \leq \kappa$, the set $X$ is a proper analytic subvariety of $\mathcal{S}$, and hence of dimension $<L$.

Proof: For our argument, it will be convenient to extend the set of allowable edge lengths from $t_{i}>0$ to a larger set including $t_{i}=0$. Once the claim is established allowing zerolength edges, we may restrict to positive-length edges (as is needed in other parts of our paper). This is simply because the original and extended parameter spaces described here have the same dimension, so the intersection of a proper analytic subvariety of the extended parameter space with the smaller parameter space must also be a proper subvariety.

Consider first the edges $e_{1}, e_{2}, e_{3}, e_{7}$ in the tree of Figure 11 In Section VI below it will be shown that when $c \leq \kappa$ there is at least one choice of a rate matrix $R$ for $\operatorname{sCov}(c, \kappa)$, and edge lengths $t_{1}>0, t_{2}=0, t_{3}=0, t_{7}>0$ so that $\exp \left(R t_{3}\right)\left(\exp \left(R t_{1}\right) J \otimes^{\text {row }} \exp \left(R t_{2}\right) J\right)$ has rank $c \kappa$ and $\exp \left(R t_{7}\right) J$ has Kruskal rank $\geq 2$. Assuming this result for now, by in addition choosing

$$
t_{9}=0, t_{8}=t_{7}, t_{6}=t_{3}, t_{5}=t_{2}, t_{4}=t_{1}
$$


we have found at least one parameter choice for $\operatorname{sCov}(c, \kappa)$ that does not lie in $X_{\mathrm{sCov}}$.

Since the same $R$ and $\left\{t_{i}\right\}$ arise from parameters for $\mathrm{eCov}(c, \kappa)$, respectively $\operatorname{Cov}(c, \kappa)$, we have also found at least one parameter choice for these models that does not lie in $X_{\mathrm{eCov}}$, respectively $X_{\mathrm{Cov}}$.

Now observe that the set of parameters for which any one of the four specified $c \kappa \times \kappa^{2}$ matrices has rank $<c \kappa$ is the zero set of a collection of analytic functions. Such functions can be explicitly constructed by composing the parameterization map for each matrix with the polynomial functions expressing the $c \kappa \times c \kappa$ minors. Similarly, the set of parameters for which a pendant edge matrix fails to have Kruskal rank $\geq 2$ is the simultaneous zero set of a collection of analytic functions built from the composition of the parameterization of that matrix with the $2 \times 2$ minors. Thus the set $X$ is the union of analytic varieties, and hence itself an analytic variety. This set cannot be the entire parameter space, since we have found one point that lies outside it. Therefore $X$ is a proper analytic subvariety, as claimed. As such, it is of dimension strictly less than $L$.

For all covarion parameters outside the set $X$ of Lemma 7 , we may apply Lemma 6 and identify $M=P_{1}^{T} \exp \left(R t_{9}\right) P_{2}$ and $\boldsymbol{\nu}=\boldsymbol{\mu} P_{1}$ for some unknown permutations $P_{1}, P_{2}$. As $X$ is of lower dimension than the parameter space, it has Lebesgue measure 0 . Thus for generic covarion parameters we may identify $M$ and $\nu$.

\section{CONSTRUCTION OF SCALED COVARION PARAMETERS WITH CERTAIN PROPERTIES}

In this section the particular parameter choice needed in the proof of Lemma 7 is constructed. We thus consider only the model sCov, with the parameters $Q, S$, and $\left\{r_{i}\right\}$ as described in Section III and $R$ as given by equation (2). We seek values of these parameters and of $t_{1}, t_{7}>0$ so that $\exp \left(R t_{1}\right) J \otimes^{\text {row }} J$ has rank $c \kappa$ and $\exp \left(R t_{7}\right) J$ has Kruskal rank at least 2 . Note that since $\exp \left(R t_{1}\right) J \otimes^{r o w} J$ is $c \kappa \times \kappa^{2}$, it may only have the desired rank when $c \leq \kappa$.

One might first consider taking $t_{1}=0$, so

$$
\exp \left(R t_{1}\right) J \otimes^{\text {row }} J=J \otimes^{\text {row }} J .
$$

However this $c \kappa \times \kappa^{2}$ matrix has rank $\kappa<c \kappa$. Similarly, taking $t_{7}=0$, so $\exp \left(R t_{7}\right) J=J$, fails to produce a matrix of Kruskal rank at least 2. Thus we must do more work to find the needed example. Our first step is to establish the following.

Lemma 8: Suppose that for each $j \in[\kappa]$, the vectors appearing as the $j$ th rows of the matrix powers $Q^{m}, m=$ $1, \ldots, c-1$ are independent. Then there exist $t_{1}, t_{7}>0$ such that $\exp \left(R t_{1}\right) J \otimes^{\text {row }} J$ has rank $c \kappa$ and $\exp \left(R t_{7}\right) J$ has Kruskal rank at least 2 .

Proof: We first show the existence of such a $t_{1}$. Let $M=M(t)=\exp (R t) J$. Because of the specific form of $J$, it is easy to see that any dependency relationship between the rows of $M \otimes^{\text {row }} J$ is equivalent to $\kappa$ separate dependency relationships between rows of $M$. Specifically, the rows of $M \otimes^{\text {row }} J$ are independent if, and only if, for each $j \in[\kappa]$ the set of the $c$ rows of $M$ with index $(i, j), i \in[c]$, are independent.
Letting $X_{j}(t)$ denote the $c \times \kappa$ submatrix of $M(t)$ consisting of the $(i, j)$ rows, we claim that some $c \times c$ minor of $X_{j}(t)$ is non-zero for all but a discrete set of values of $t$. Since there are only finitely many $j$ to consider, this implies the existence of the desired $t_{1}$.

Fixing $j$, for notational ease let

$$
X_{j}(t)=\left(\begin{array}{c}
\mathbf{x}_{1}(t) \\
\vdots \\
\mathbf{x}_{c}(t)
\end{array}\right), \quad x(t)=\operatorname{det}\left(\begin{array}{c}
\overline{\mathbf{x}}_{1}(t) \\
\vdots \\
\overline{\mathbf{x}}_{c}(t)
\end{array}\right)
$$

where the bar denotes projection onto some choice of $c$ coordinates, to be specified later, so that $x(t)$ is a specific $c \times c$ minor of $X_{j}(t)$.

Since $x(t)$ is an analytic function, to establish that it is nonzero except at a discrete set of points, it is enough to show it is not identically zero. Now $x(t)$ is easily evaluated only at $t=0$, and unfortunately $x(0)=0$ since $\mathbf{x}_{i}(0)$ is the standard basis vector $\mathbf{e}_{j}$ for all $i$. We will, however, show $x(t)$ is not identically zero by showing the derivative $x^{(n)}(0)$ is non-zero for $n=c(c-1) / 2$.

To obtain information on the derivatives $\mathbf{x}_{i}^{(l)}(0)$, observe that $M(t)$ is the solution to the initial value problem $M^{\prime}=$ $R M, M(0)=J$. Thus $\mathbf{x}_{i}^{(l)}(0)$ is the $(i, j)$ row of $R^{l} J$. Moreover, since $S \mathbf{1}_{c}^{T}=\mathbf{0}$,

$$
\begin{aligned}
R^{l} J & =\left(\operatorname{diag}\left(r_{1}, r_{2}, \ldots, r_{c}\right) \otimes Q+S \otimes I_{\kappa}\right)^{l}\left(\mathbf{1}_{c}^{T} \otimes I_{\kappa}\right) \\
& =\operatorname{diag}\left(r_{1}, r_{2}, \ldots, r_{c}\right)^{l} \mathbf{1}_{c}^{T} \otimes Q^{l}+\sum_{m=1}^{l-1} \mathbf{y}_{l, m}^{T} \otimes Q^{m},
\end{aligned}
$$

for some vectors $\mathbf{y}_{l, m}$. Thus, for $l \geq 1, \mathbf{x}_{i}^{(l)}(0)$ is a linear combination of the $j$ th rows of $Q^{m}, 1 \leq m \leq l$, where the $j$ th row of $Q^{l}$ appears with coefficient $r_{i}^{l}$.

Now with $n=c(c-1) / 2$,

$$
x^{(n)}(0)=\sum_{\lambda=\left(n_{1}, \ldots, n_{c}\right)} m_{\lambda} \operatorname{det}\left(\overline{\mathbf{x}}_{1}^{\left(n_{1}\right)}(0), \ldots, \overline{\mathbf{x}}_{c}^{\left(n_{c}\right)}(0)\right) \text {, }
$$

where the summation is over non-negative integer solutions to $n_{1}+\cdots+n_{c}=n$ and $m_{\lambda}=\left(\begin{array}{c}n \\ n_{1}, \ldots, n_{c}\end{array}\right)$ is a multinomial coefficient. Letting $\mathbf{z}_{0}=\mathbf{e}_{j}$ and $\mathbf{z}_{i}$ be the $j$ th row of $Q^{i}$ for $i \geq 1$, we have shown that $\mathbf{x}_{i}^{(l)}(0)$ lies in the span of $\left\{\mathbf{z}_{i}\right\}_{i=0}^{l}$ for all $l \geq 0$. This implies that any summand in equation (3) must vanish if more than $l+1$ of the $n_{i}$ satisfy $n_{i} \leq l$, since in that case the rows in the determinant are dependent. But $n=c(c-1) / 2=0+1+\cdots+(c-1)$, hence non-zero terms can arise only when $\lambda$ is a permutation of $(0,1, \ldots, c-1)$.

With $S_{c}$ denoting the permutations of $(1, \ldots, c)$, and $m=$ $m_{(0,1, \ldots, c-1)}$,

$$
\begin{aligned}
x^{(n)}(0) & =m \sum_{\mu \in S_{c}} \operatorname{det}\left(\overline{\mathbf{x}}_{1}^{\left(\mu^{-1}(1)-1\right)}(0), \ldots, \overline{\mathbf{x}}_{c}^{\left(\mu^{-1}(c)-1\right)}(0)\right) \\
& =m \sum_{\mu \in S_{c}} \operatorname{sgn}(\mu) \operatorname{det}\left(\overline{\mathbf{x}}_{\mu(1)}^{(0)}(0), \ldots, \overline{\mathbf{x}}_{\mu(c)}^{(c-1)}(0)\right) .
\end{aligned}
$$


But with $Z=\left(\mathbf{z}_{0}^{T}, \ldots, \mathbf{z}_{c-1}^{T}\right)^{T}$, we have shown

$$
\left(\begin{array}{c}
\mathbf{x}_{\mu(1)}^{(0)}(0) \\
\vdots \\
\mathbf{x}_{\mu(c)}^{(c-1)}(0)
\end{array}\right)=L_{\mu} Z
$$

where $L_{\mu}$ is a $c \times c$ lower triangular matrix with diagonal entries $L_{i, i}=r_{\mu(i)}^{i-1}$. By hypothesis, all rows of $Z$ except the first form an independent set, and since $Q^{l} \mathbf{1}_{c}^{T}=\mathbf{0}$ for $l \geq$ 1 while $\mathbf{z}_{0} \mathbf{1}_{c}^{T}=1$, the first row is not in the span of the others. Thus $Z$ has rank $c$, and some choice of $c$ of its columns are independent. Specifying that the bar over a matrix or row vector designates a projection onto these column coordinates yields

$$
\left(\begin{array}{c}
\overline{\mathbf{x}}_{\mu(1)}^{(0)}(0) \\
\vdots \\
\overline{\mathbf{x}}_{\mu(c)}^{(c-1)}(0)
\end{array}\right)=L_{\mu} \bar{Z},
$$

so

$$
\operatorname{det}\left(\overline{\mathbf{x}}_{\mu(1)}^{(0)}(0), \ldots, \overline{\mathbf{x}}_{\mu(c)}^{(c-1)}(0)\right)=\left(\prod_{i=1}^{c} r_{\mu(i)}^{i-1}\right) \operatorname{det}(\bar{Z}) .
$$

Since $\operatorname{det}(\bar{Z}) \neq 0$, to see that $x^{(n)}(0) \neq 0$ it is enough to show

$$
\sum_{\mu \in S_{c}} \operatorname{sgn}(\mu) \prod_{i=1}^{c} r_{\mu(i)}^{i-1} \neq 0
$$

But the left hand side is a Vandermonde determinant, and since the $r_{i}$ are distinct, it does not vanish. Thus the desired $t_{1}$ exists.

For the existence of $t_{7}$, consider the $\left(i_{1}, j_{1}\right)$ and $\left(i_{2}, j_{2}\right)$ rows of $\exp (R t) J$. If $j_{1} \neq j_{2}$, then these rows are independent when $t=0$, hence for all $t$ except a discrete set. If $j_{1}=j_{2}$, then the two rows are rows of $X_{j_{1}}(t)$, and thus independent for all but a discrete set of $t$ by our work above. Since there are only finitely many pairs to consider, for all but a discrete set of values we find $\exp (R t) J$ has Kruskal rank $\geq 2$.

The existence of rate matrices $Q$ satisfying the hypotheses of the last lemma is a consequence of the following one.

Lemma 9: Suppose a $\kappa \times \kappa$ rate matrix $Q$ has at least $c$ distinct eigenvalues and its right eigenvectors can be chosen to have all non-zero entries. Then for each $j \in[\kappa]$ the vectors appearing as the $j$ th rows of $Q^{l}, l=0, \ldots, c-1$, are independent.

Proof: Let $Q=U D U^{-1}$ be a diagonalization of $Q$. Then with $\mathbf{u}_{j}$ denoting the $j$ th row of $U$, the $j$ th row of $Q^{l}$ is $\mathbf{u}_{j} D^{l} U^{-1}$. To show these rows are independent, it is enough to show the $\mathbf{u}_{j} D^{l}, l=0, \ldots, c-1$ are independent, or even that the projections of these vectors onto some choice of $c$ coordinates are independent. By choosing to project onto $c$ coordinates corresponding to distinct diagonal entries of $D$, we may reduce to the case where $D$ is $c \times c$ with distinct diagonal entries and the vectors $\mathbf{u}_{j} \in \mathbb{C}^{c}$ have all non-zero entries.

But if $W$ is the $c \times c$ matrix whose $l$ th row is $\mathbf{u}_{j} D^{l-1}$, then $W=V \operatorname{diag}\left(\mathbf{u}_{j}\right)$ where $V$ is a Vandermonde matrix constructed from the diagonal entries of $D$. By our assumptions, both $V$ and $\operatorname{diag}\left(\mathbf{u}_{j}\right)$ have non-zero determinants, so $W$ does as well. Thus the rows of $W$ are independent.

To see a $Q$ satisfying the hypotheses of Lemma 9 exists, let

$$
Q_{0}=\frac{1}{\kappa(\kappa-1)}\left(\mathbf{1}_{\kappa}^{T} \mathbf{1}_{\kappa}-\kappa I_{\kappa}\right)
$$

be a generalized Jukes-Cantor matrix of size $\kappa$, all of whose off-diagonal entries are equal, which has stationary vector $\mathbf{1}_{\kappa}$. The eigenspaces of $Q_{0}$ are the span of $\mathbf{1}_{\kappa}$ and its orthogonal complement. For a diagonalization $Q_{0}=U D_{0} U^{-1}$ we can thus chose $U$ to be an orthogonal matrix all of whose entries are non-zero. (For instance, when $\kappa=4$ we may choose $U$ to be a Hadamard matrix.) Since $D_{0}$ has repeated diagonal entries, perturb the non-zero entries slightly to obtain a diagonal matrix $D$ without repetitions, and let $Q=U D U^{-1}$. Since $Q$ also has $\mathbf{1}_{\kappa}$ as its stationary distribution, and since $Q$ is symmetric, it is a rate matrix of the sort needed.

Choosing such a $Q$ and any $S$ and distinct $r_{i}$ for the sCov parameters gives a particular choice of scaled covarion parameters $Q, S,\left\{r_{i}\right\}$ such that there exists a $t_{1}>0$ where $\exp \left(R t_{1}\right) J \otimes J$ has rank $c \kappa$, and a $t_{7} \geq 0$ such that $\exp \left(R t_{7}\right) J$ has Kruskal rank at least 2.

Thus Lemma 7 is fully established.

\section{IDENTIFYING THE COVARION RATE MATRIX $R$}

The next goal is to use $\boldsymbol{\nu}=\boldsymbol{\mu} P_{1}$ and $M=P_{1}^{T} \exp \left(R t_{9}\right) P_{2}$, as identified in Section $\mathrm{V}$ through Lemmas 6 and 7 to determine the covarion root distribution $\boldsymbol{\mu}$ and the covarion rate matrix $R$. It is of course enough to determine $R t_{9}$, where $t_{9}>0$ is the edge length, and then use the required normalization of $R$.

Let us assume $\boldsymbol{\nu}$ has its entries in non-increasing order. (This can be achieved by multiplying $\nu$ on the right by some permutation $P$, and $M$ on the left by $P^{T}$, thereby changing the unknown $P_{1}$.) Now since $\operatorname{diag}(\boldsymbol{\mu}) \exp (R t)$ is symmetric, and $\operatorname{diag}(\boldsymbol{\nu})=P_{1}^{T} \operatorname{diag}(\boldsymbol{\mu}) P_{1}$, one can verify that $\operatorname{diag}(\boldsymbol{\nu}) M P_{2}^{T} P_{1}$ is symmetric as well. This shows there is at least one reordering of the columns of $M$ that results in $\operatorname{diag}(\boldsymbol{\nu}) M$ being symmetric. Assume some such ordering of the columns of $M$ has been chosen to ensure this symmetry.

If $\boldsymbol{\nu}$ (equivalently, $\boldsymbol{\mu}$ ) has no repeated entries, these choices have uniquely determined an ordering to the rows and columns of $M$, and forced $P_{2}=P_{1}$. To see this, note the rows of $M$ have a fixed correspondence to entries of $\nu$, which have a unique decreasing ordering. For the columns, note that the symmetry of $\operatorname{diag}(\boldsymbol{\nu}) M$ and the fact that $\mathbf{1}_{c \kappa} M^{T}=\mathbf{1}_{c \kappa}$ implies $\boldsymbol{\nu} M=\boldsymbol{\nu}$. However, if the columns of $M$ are permuted by $P$, then $\boldsymbol{\nu} M P=\boldsymbol{\nu} P \neq \boldsymbol{\nu}$. We therefore can conclude $\boldsymbol{\nu}=\boldsymbol{\mu} P_{1}$ and $M=P_{1}^{T} \exp \left(R t_{9}\right) P_{1}$ for some unknown permutation $P_{1}$.

Since $\boldsymbol{\nu}$ may have repeated entries, the above argument only holds for generic choices of parameters. In order to avoid introducing any generic conditions other than those already arising from the application of Kruskal's theorem, we give an alternate argument using the following lemma.

Lemma 10: Suppose that a matrix $M$ has a factorization of the form $M=P W^{T} Z W$ for some real symmetric positivedefinite $m \times m$ matrix $Z$, real $m \times n$ matrix $W$ of rank $n$, 
and $n \times n$ permutation $P$. Then $P$ is uniquely determined by $M$.

Proof: The matrix $Z$ defines an inner product on $\mathbb{R}^{m}$, and if $\boldsymbol{w}_{i}$ denotes the $i$ th column of $W$, then the $i, j$ entry of the symmetric matrix $N=W^{T} Z W$ is

$$
\left\langle\boldsymbol{w}_{i}, \boldsymbol{w}_{j}\right\rangle_{Z}=\boldsymbol{w}_{i}^{T} Z \boldsymbol{w}_{j}
$$

But for any inner product, if $\boldsymbol{x} \neq \boldsymbol{y}$ then

$$
\langle\boldsymbol{x}, \boldsymbol{x}\rangle+\langle\boldsymbol{y}, \boldsymbol{y}\rangle>2\langle\boldsymbol{x}, \boldsymbol{y}\rangle .
$$

Now the matrix $W$ has distinct columns since it has rank $n$. Thus the entries of $N$ satisfy

$$
n_{i i}+n_{j j}>2 n_{i j}
$$

Suppose for some permutations $P_{1}, P_{2}$ the matrices $N_{1}=$ $P_{1}^{T} M$ and $N_{2}=P_{2}^{T} M$ are both symmetric, and have entries satisfying the inequalities (4). Note also that $N_{1}$ and $N_{2}$ have the same set of rows.

Consider first the largest entry (or entries, in case of ties) of $N_{1}$ and $N_{2}$. Because the inequality in (4) is strict, a largest entry cannot appear off the diagonal. Thus the row (or rows) of $N_{1}$ and $N_{2}$ containing the largest entry (or entries) must occur in the same positions. Since the same argument applies to the submatrices obtained from the $N_{i}$ by deleting the rows and columns with the largest entries, repeated application shows $N_{1}=N_{2}$. Thus $P_{1}=P_{2}$.

Corollary 11: Suppose $\nu, M$ are of the form

$$
\boldsymbol{\nu}=\boldsymbol{\mu} P_{1}, \quad M=P_{1}^{T} \exp (R t) P_{2},
$$

for some covarion rate matrix $R$ with stationary vector $\boldsymbol{\mu}$, permutations $P_{1}, P_{2}$, and scalar $t$. Then $P_{1}^{T} P_{2}$ is uniquely determined.

Proof: Apply Lemma 10 to $\operatorname{diag}(\boldsymbol{\nu}) M$, with $P=P_{1}^{T} P_{2}$, $W=P_{2}$, and $Z=\operatorname{diag}(\boldsymbol{\mu}) \exp (R t)$.

As a consequence of this corollary, after multiplying $M$ on the right by $\left(P_{1}^{T} P_{2}\right)^{T}$ we may now assume we have

$$
\boldsymbol{\nu}=\boldsymbol{\mu} P, \quad M=P^{T} \exp (R t) P
$$

for some (unknown) permutation $P$. But then $M=$ $\exp \left(P^{T} R P t\right)$, and since this matrix is diagonalizable with positive eigenvalues, $P^{T} R P t$ is determined by applying the logarithm to its diagonalization.

Now $P^{T} R P t$ is simply a rescaled version of $R$ with the same permutation applied to rows and columns. Thus there exists at least one simultaneous permutation of the rows and columns of $P^{T} R P t$ which yields a rescaled covarion rate matrix. However, we do not yet know if there is a unique such permutation, or a unique such covarion rate matrix.

One might suspect that the pattern of zero entries in the off-diagonal blocks of a covarion rate matrix should allow the (almost) unique determination of $R t$ from this permuted form. This is the content of the following lemma.

Lemma 12: Let $R_{1}, R_{2}$ be rate matrices for $\operatorname{Cov}(c, \kappa)$, with $R_{1}$ class irreducible, as defined in Section [I] Suppose for permutations $P_{1}, P_{2}$, and scalars $t_{1}, t_{2}>0$, that

$$
P_{1}^{T} R_{1} P_{1} t_{1}=P_{2}^{T} R_{2} P_{2} t_{2}
$$

If $c \neq \kappa$ then $t_{1}=t_{2}$, and $P=P_{1} P_{2}^{T}$ can be expressed as $P=\widehat{P} \otimes \widetilde{P}$ for some $c \times c$ permutation $\widehat{P}$ and $\kappa \times \kappa$ permutation $\widetilde{P}$. Thus $R_{1}$ can be determined up to application of a permutation of the form $\widehat{P} \otimes \widetilde{P}$.

If $R_{1}, R_{2}$ are rate matrices for either $\operatorname{sCov}(c, \kappa)$ or $\mathrm{eCov}(c, \kappa)$, then the same result holds for all $c$.

Note that a permutation of the form $\widehat{P} \otimes \widetilde{P}$ can be viewed as a permutation of classes by $\widehat{P}$, and a simultaneous permutation of bases within all classes by $\widetilde{P}$.

Proof: Using the normalization of $R_{1}$ and $R_{2}$, it is trivial to see that $t_{1}=t_{2}$. Conjugating by $P_{2}$, we obtain $P^{T} R_{1} P=$ $R_{2}$.

Let $N$ be a matrix of the same size as $R_{1}$, with entry 1 (respectively, 0 ) wherever the corresponding entry of $R_{1}$ is positive (respectively non-positive). Let $G_{1}=G\left(R_{1}\right)$ be the (undirected) graph whose adjacency matrix is $N=N^{T}$. Thus the vertices of $G_{1}$ are labeled by the elements of $[c] \times[\kappa]$, the indices corresponding to rows and columns of $R_{1}$, and an edge joins vertices $i$ and $j$ exactly when $R_{1}(i, j)>0$ (or, equivalently, when $\left.R_{1}(j, i)>0\right)$. $G_{1}$ is the 'communication graph' of $R_{1}$, expressing which instantaneous state changes can occur.

By assumptions on $R_{1}$, for each class $i$ with $Q_{i} \neq 0$, the vertices labeled $(i, j), j \in[\kappa]$, corresponding to all states in class $i$, form a clique (i.e., the subgraph on these vertices is a complete graph) of size $\kappa$. Moreover, these cliques are each maximal, since any vertex $\left(i^{\prime}, j^{\prime}\right)$ outside of the clique has $i^{\prime} \neq i$ and is connected to at most one vertex in the clique, namely $\left(i, j^{\prime}\right)$, which has the same base but different class.

Suppose first that $c \neq \kappa$. In this case we show there are no other maximal cliques of size $\kappa$. To this end, suppose a vertex labeled $(i, j)$ is in some other maximal clique $\mathcal{C}$ of size $\kappa$. The only vertices adjacent to it outside of its class correspond to the same base $j$. Thus $\mathcal{C}$ must contain at least one of these, say $(k, j)$ where $k \neq i$. As the $(k, j)$ vertex and any $(i, l)$ vertex cannot be in a common clique if $j \neq l, \mathcal{C}$ must contain only vertices corresponding to base $j$. As there are $c \neq \kappa$ of these, they cannot form a clique of size $\kappa$.

Now if we similarly construct $G_{2}=G\left(R_{2}\right)$, the statement $P^{T} R_{1} P=R_{2}$ means there is a graph isomorphism from $G_{1}$ to $G_{2}$, obtained by relabeling vertices according to the permutation $P$. As such an isomorphism must take maximal cliques to maximal cliques, we see that $P$ must map all states in an $R_{1}$ class with $Q_{i} \neq 0$ to all states in an $R_{2}$ class with $Q_{j} \neq 0$. (As the covarion model allows at most one class with $Q_{i}=0$, this also means that if either $R_{i}$ has a class with $Q_{i}=0$, then so does the other, and these classes must also be mapped to one another.)

This implies $P$ has the following structure: Partition $P$ into a $c \times c$ matrix of $\kappa \times \kappa$ blocks, corresponding to classes. All blocks of $P$ are zero, except for one block in each row and column. Let $\widehat{P}$ be the $c \times c$ permutation matrix with $1 \mathrm{~s}$ in positions corresponding to those non-zero blocks. The nonzero blocks of $P$ are also $\kappa \times \kappa$ permutation matrices.

We next claim that the non-zero $\kappa \times \kappa$ blocks in $P$ are all identical. To see this, consider how $P$ acts on a non-zero off-diagonal block $S_{i_{1} i_{2}}$ of $R_{1}$ through the formula $P^{T} R_{1} P$ : 
the resulting block has the form $\widetilde{P}_{1}^{T} S_{i_{1} i_{2}} \widetilde{P}_{2}$ where $\widetilde{P}_{1}$ and $\widetilde{P}_{2}$ are two of the $\kappa \times \kappa$ permutations appearing as blocks of $P$. But this must equal the corresponding block of $R_{2}$, which is diagonal. Thus if all diagonal entries of $S_{i_{1} i_{2}}$ are non-zero then $\widetilde{P}_{1}^{T} \widetilde{P}_{2}=I_{\kappa}$, so $\widetilde{P}_{1}=\widetilde{P}_{2}$. The class irreducibility of $R_{1}$ ensures that we obtain enough such equalities to see that all $\widetilde{P}_{i}$ are equal to some common $\kappa \times \kappa$ permutation $\widetilde{P}$. Thus $P=\widehat{P} \otimes \widetilde{P}$.

Now for the models sCov and eCov consider the case of $c=$ $\kappa$. In this case, maximal cliques corresponding to either a fixed base or a fixed class have the same cardinality, but there can be no other maximal cliques. Unless the graph isomorphism from $G_{1}$ to $G_{2}$ maps some fixed-base clique to a fixed-class clique, our earlier argument applies.

We therefore suppose that the base $j$ clique is mapped to the class $i$ clique, and argue toward a contradiction. This means $P$ maps vertices in $G_{1}$ labeled $(k, j)$ for $k=1, \ldots, c$ to vertices labeled $(i, l)$ for $l=1, \ldots, \kappa$ in $G_{2}$. As a result, every other fixed-base clique in $G_{1}$ must also map to a fixed-class clique in $G_{2}$, since all the fixed-base cliques of $G_{2}$ include some $(i, l)$.

But the formula $P^{T} R_{1} P=R_{2}$ implies that each diagonal block of $R_{2}$ must have as its $\kappa^{2}-\kappa$ off-diagonal entries the $\kappa^{2}-\kappa$ values $s_{i_{1} i_{2}} \neq 0$ which appear in the off-diagonal blocks of $R_{1}$. But this is impossible, since the base-change matrices $Q_{i}$ of $R_{2}$ are assumed not to be equal.

We now have determined $R$ and $\boldsymbol{\mu}$ up to separate permutations $\widetilde{P}$ of the bases and $\widehat{P}$ of the classes. The ambiguity expressed by $\widehat{P}$ cannot be removed, as permuting classes has no effect on the distributions defined by the model. Our next step is to use information on the ordering of the bases obtained at the leaves of the tree in order to determine $\widetilde{P}$.

Let $P^{T} M_{7}$ denote the $c \kappa \times \kappa$ matrix, which was determined via Lemma 6, describing permuted transition probabilities on edge $e_{7}$ of the tree of Figure 1 . Assuming $P=\widehat{P} \otimes \widetilde{P}$ by previous steps in our analysis, $(\widehat{P} \otimes \widetilde{P})^{T} \exp \left(R t_{7}\right) J$ is known.

Lemma 13: Suppose $W=P^{T} \exp (R t) J$ for some permutation $P=\widehat{P} \otimes \widetilde{P}$, covarion rate matrix $R$, and scalar $t$. Then $\widetilde{P}$ is uniquely determined.

Proof: Consider the $\kappa \times \kappa$ matrix, determined by known information,

$$
\begin{aligned}
J^{T} \operatorname{diag}(\boldsymbol{\nu}) W & =J^{T} P^{T} \operatorname{diag}(\boldsymbol{\mu}) P P^{T} \exp \left(R t_{7}\right) J \\
& =\left(\mathbf{1}_{c} \otimes I_{\kappa}\right)\left(\widehat{P}^{T} \otimes \widetilde{P}^{T}\right) \operatorname{diag}(\boldsymbol{\mu}) \exp \left(R t_{7}\right) J \\
& =\left(\mathbf{1}_{c} \widehat{P}^{T} \otimes I_{\kappa} \widetilde{P}^{T}\right) \operatorname{diag}(\boldsymbol{\mu}) \exp \left(R t_{7}\right) J \\
& =\left(\mathbf{1}_{c} \otimes \widetilde{P}^{T}\right) \operatorname{diag}(\boldsymbol{\mu}) \exp \left(R t_{7}\right) J \\
& =\widetilde{P}^{T}\left(\mathbf{1}_{c} \otimes I_{\kappa}\right) \operatorname{diag}(\boldsymbol{\mu}) \exp \left(R t_{7}\right) J \\
& =\widetilde{P}^{T} N
\end{aligned}
$$

where $N=J^{T} \operatorname{diag}(\boldsymbol{\mu}) \exp \left(R t_{7}\right) J$. From Lemma 3, we also have that

$$
N=K^{T} \exp \left(B t_{7}\right) K
$$

where $B$ is real diagonal and $K$ has rank $\kappa$. We may thus apply Lemma 10 to the product

$$
J^{T} \operatorname{diag}(\nu) W=\widetilde{P}^{T} K^{T} \exp \left(B t_{7}\right) K
$$

to determine $\widetilde{P}$.

Thus for generic parameters, $R$ and $\boldsymbol{\mu}$ are determined uniquely, up to the permutation $\widehat{P}$ of classes.

Remark 2: That the restriction $c<\kappa$ is necessary for the Cov model in Lemma 12 can be easily seen. For example, with $\kappa=c=2$, the two rate matrices

$$
\begin{aligned}
R & =\frac{1}{14}\left(\begin{array}{cccc}
-5 & 3 & 2 & 0 \\
3 & -4 & 0 & 1 \\
2 & 0 & -3 & 1 \\
0 & 1 & 1 & -2
\end{array}\right), \\
R^{\prime} & =\frac{1}{14}\left(\begin{array}{cccc}
-5 & 2 & 3 & 0 \\
2 & -3 & 0 & 1 \\
3 & 0 & -4 & 1 \\
0 & 1 & 1 & -2
\end{array}\right)
\end{aligned}
$$

are related by exchanging rates and classes. Note further that both $R$ and $R^{\prime}$ have $\frac{1}{4} \mathbf{1}_{4}$ as their stationary distribution, so they lead to the same observed distribution at a single leaf. Moreover, they lead to the same set of observable distributions at two leaves when one considers all possible edge lengths $t \geq 0$. Thus one cannot use the observed distribution at one or two leaves to distinguish between distributions arising from these two rate matrices.

Of course one might next attempt to use observed joint distributions at multiple leaves to distinguish these parameters, or introduce additional generic conditions to obtain identifiability of numerical Cov parameters even when $c=\kappa$. As we have not pursued these directions, we do not claim identifiability fails for generic parameters in this case, but only that the arguments given above do not establish it.

\section{IDENTIFYING EDGE LENGTHS}

As $R$ is now known, all that remains is to determine edge lengths. By simple and well-known arguments [18], these can be determined from knowing total distances between leaves of the tree. Thus the determination of all edge lengths is established by the following.

Lemma 14: Fix a covarion rate matrix $R$, of size $c \kappa \times c \kappa$. Suppose a $\kappa \times \kappa$ matrix $N$ is in the image of the resulting covarion model on a 2-taxon tree, with edge length $t$. Then $N$ uniquely determines $t$.

Proof: From Lemma 3, we have that

$$
N=K^{T} \exp (B t) K,
$$

where $B=\operatorname{diag}\left(\beta_{1}, \ldots, \beta_{c \kappa}\right), 0=\beta_{1}>\beta_{2} \geq \cdots \geq \beta_{c \kappa}$ and $K$ is a real $c \kappa \times \kappa$ matrix, of rank $\kappa$. Furthermore, since $R$ is known, so are all $\beta_{i}$ and $K$.

With $K=\left(k_{j i}\right)$ and $N=\left(n_{i j}\right)$, this implies the diagonal entries of $N$ are

$$
n_{i i}=\sum_{j=1}^{c \kappa} k_{j i}^{2} \exp \left(\beta_{j} t\right) .
$$

As the $k_{j i}$ are real numbers and all $\beta_{i}$ are non-positive, each term in this formula is a non-increasing function of $t$. Thus $n_{i i}=n_{i i}(t)$ is a non-increasing function of $t$. If we show that for some $i$ the function $n_{i i}(t)$ is strictly decreasing, then from any value of $n_{i i}$ we may determine $t$. But to establish 
that some $n_{i i}$ is strictly decreasing, we need only show there exists some $i$ and some $j>1$ such that $k_{j i} \neq 0$, so that at least one term in equation (5) is a strictly decreasing function. However, as $K$ has rank $\kappa>1$, we cannot have $k_{j i}=0$ for all $j>1$.

\section{REFERENCES}

[1] Elizabeth S. Allman, Cécile Ané, and John A. Rhodes. Identifiability of a Markovian model of molecular evolution with gammadistributed rates. Advances in Applied Probability, 40(1):229-249, 2008. arXiv:0709.0531.

[2] Elizabeth S. Allman and John A. Rhodes. The identifiability of tree topology for phylogenetic models, including covarion and mixture models. J. Comput. Biol., 13(5):1101-1113, 2006. arXiv:q-bio.PE/0511009.

[3] Elizabeth S. Allman and John A. Rhodes. Identifying evolutionary trees and substitution parameters for the general Markov model with invariable sites. Math. Biosci., 211(1):18-33, 2008. arXiv:q-bio.PE/0702050.

[4] Elizabeth S. Allman and John A. Rhodes. Phylogenetic ideals and varieties for the general Markov model. Adv. in Appl. Math., 40(2), 2008. arXiv:math. AG/0410604.

[5] Joseph T. Chang. Full reconstruction of Markov models on evolutionary trees: identifiability and consistency. Math. Biosci., 137(1):51-73, 1996.

[6] Walter M. Fitch and Etan Markowitz. An improved method for determining codon variability in a gene and its application to the rate of fixation of mutations in evolution. Biochemical Genetics, 4:579-593, 1970 .

[7] Nicolas Galtier. Maximum-likelihood phylogenetic analysis under a covarion-like model. Mol. Biol. Evol., 18(5):866-873, 2001.

[8] Nicolas Galtier and A. Jean-Marie. Markov-modulated Markov chains and the covarion process of of molecular evolution. J. Comput. Biol., 11(4):727-733, 2004.

[9] Olivier Gascuel and Stéphane Guindon. Modelling the variability of evolutionary processes. In Olivier Gascuel and Mike Steel, editors, Reconstructing Evolution: New Mathematical and Computational Advances, pages 65-107. Oxford University Press, 2007.

[10] Stéphane Guindon, Allen G. Rodrigo, Kelly A. Dyer, and John P. Huelsenbeck. Modeling the site-specific variation of selection patterns across lineages. P.N.A.S., 101:12957-12962, 2004.

[11] Roger A. Horn and Charles R. Johnson. Matrix Analysis. Cambridge University Press, 1985.

[12] John Huelsenbeck. Testing a covariotide model of DNA substitution. Mol. Biol. Evol., 19:698-707, 2002.

[13] J. B. Kruskal. Rank, decomposition, and uniqueness for 3-way and $N$-way arrays. In Multiway data analysis (Rome, 1988), pages 7-18. North-Holland, Amsterdam, 1989.

[14] Joseph B. Kruskal. More factors than subjects, tests and treatments: an indeterminacy theorem for canonical decomposition and individual differences scaling. Psychometrika, 41(3):281-293, 1976.

[15] Joseph B. Kruskal. Three-way arrays: rank and uniqueness of trilinear decompositions, with application to arithmetic complexity and statistics. Linear Algebra and Appl., 18(2):95-138, 1977.

[16] T. Petrie. Probabilistic functions of finite state Markov chains. Ann. Math. Statist, 40:97-115, 1969.

[17] James S. Rogers. Maximum likelihood estimation of phylogenetic trees is consistent when substitution rates vary according to the invariable sites plus gamma distribution. Syst. Biol., 50(5):713-722, 2001.

[18] Charles Semple and Mike Steel. Phylogenetics, volume 24 of Oxford Lecture Series in Mathematics and its Applications. Oxford University Press, Oxford, 2003.

[19] Chris Tuffley and Mike Steel. Modeling the covarion hypothesis of nucleotide substitution. Math. Biosci., 147(1):63-91, 1998.

[20] Huai-Chun Wang, Matthew Spencer, Edward Susko, and Andrew Roger Testing for covarion-like evolution in protein sequences. Mol. Biol. Evol., 24(1):294-305, 2007.

[21] Simon Whelan. Spatial and temporal heterogeneity in nucleotide evolution. preprint, (2008). 